\title{
Numerical techniques for stochastic foot and mouth disease epidemic model with the impact of vaccination
}

\author{
Kamaleldin Abodayeh ${ }^{* *}$ (D, Muhammad Shoaib Arif ${ }^{2}$, Ali Raza ${ }^{2}$, Muhammad Rafiq $^{3}$, Mairaj Bibi ${ }^{4}$ and \\ Amna Nazeer ${ }^{4}$
}

"Correspondence: kamal@psu.edu.sa ${ }^{1}$ Department of Mathematics and General Sciences, Prince Sultan University, Riyadh, Saudi Arabia Full list of author information is available at the end of the article

\section{Springer}

\begin{abstract}
The spread of an epidemic diseases is stochastic in nature. It is more realistic to include this stochasticity when modelling the dynamics of a communicable disease. In this paper, a stochastic model for foot and mouth disease dynamics in animals is constructed from its corresponding deterministic model. Like the deterministic model, the dynamics of the stochastic model is also governed by a threshold parameter $A^{*}$, called FMD generation number. If we are able to make $A^{*}<1$, then the disease will completely die out from the animal population. If $A^{*}>1$, the disease will become endemic in animals. A competitive structure preserving numerical analysis of the stochastic model in comparison with its deterministic part is presented. The proposed numerical analysis is also compared with already existing numerical techniques which may not be reliable in certain situations. Numerical experiments are performed and their results are plotted to show the strength of the proposed technique.
\end{abstract}

Keywords: Foot and mouth disease (FMD); Stochastic numerical techniques; Stability

\section{Literature survey}

It was in Venice in 1514 that an Italian monk Hieronymus Fracastorius first explained the possible foot-and-mouth disease among cattle. The victims of this disease could not eat, and their mouths, feet had vesicles. These five hundred years-old descriptions of the FMD disease have close similarity with the present state of the same disease. This disease is deemed to be the most common one among split hoof animals, as it affects seventy species of split hoof animals such as goats and sheep. The disease was ubiquitous in areas where livestock used to be kept. The disease still exists in more than one hundred countries, and its spread reflects economic progress. Although most of the countries have eradicated this disease, its spread in new countries, which were previously disease-free, could result in huge financial losses. A single stood positive-sense RNA virus is responsible for this disease known as foot and mouth disease virus. The FMD virus known as aphthous virus from the family of picornaviridae. The present article is about the description of the virus

(c) The Author(s) 2020. This article is licensed under a Creative Commons Attribution 4.0 International License, which permits use sharing, adaptation, distribution and reproduction in any medium or format, as long as you give appropriate credit to the original author(s) and the source, provide a link to the Creative Commons licence, and indicate if changes were made. The images or other third party material in this article are included in the article's Creative Commons licence, unless indicated otherwise in a credit line to the material. If material is not included in the article's Creative Commons licence and your intended use is not permitted by statutory regulation or exceeds the permitted use, you will need to obtain permission directly from the copyright holder. To view a copy of this licence, visit http://creativecommons.org/licenses/by/4.0/. 
and the classification of FMD outbreaks. This information would help explain the present existence of the disease all over the world. How could the FMD progressive control pathway help in checking the spread of disease in affected countries? European region is given central attention in the study of FMD virus $[3,4,8]$.

In 2000, the O-Pan Asia virus was detected in Uzbekistan, Mongolia, Armenia, Georgia, Russia, Kyrgyzstan, and Tajikistan. The virus spread to the province of KwaZulu-Natal in South Africa. It was the first reported eruption in that country because of serotype $\mathrm{O}$ in 1957. The eruption was connected with the fact that the pigs were fed on swill that came from Asia. On the other hand, eruptions caused by SAT1 were recorded once again in 2013. In 2001, the eruption of FMD disease occurred in England. However, it was attached by a powerful eruption of FMD disease in 1967, and approximately four million cattle were culled in order to eliminate the disease [5].

The eruption of disease in 2001 was checked by resorting to a stamping out strategy in which approximately six million affected cattle were destroyed. The rough estimate of the total economic loss was approximately twelve or thirteen billion US dollar. At present, the Pan Asia-II strain has caused an eruption of disease at a large scale in the whole Middle East and south Asian regions. In 2009, this strain was also blamed for FMD in Pakistan that extended to the west and became the cause of disease in Turkey, Israel, Libya, and Bulgaria. The latter suffered through an eruption of disease of FMD at the end of 2010 and the first case of the disease was discovered in a wild boar. The FMD virus, serotype $\mathrm{O}$ virus, and strain of Pan Asia-II were identified to be the cause of eruption of disease in eleven different places. Buffalo, sheep, and goats can be directly affected by the FMD virus. A stamping out policy was resorted to, and approximately one thousand cattle were culled. Seropositive wildlife was identified in neighbouring areas where the eruption of disease happened. Most counties were labelled as free of FMD in July 2011. But the FMD virus still persists and affects the economy of countries [6]. By the utilization of numerous mathematical structures, we are able to have a sound perception of widespread diseases. We can analyze susceptibility and contact of conjuncture model by model formulation and appropriate imitation. The results from a considerable effect on the health of animals by estimating the mediator, host, and environmental conditions and scientifically authentic outcomes can be drawn by the persons assigned to the task of policymaking on health and can finally be converted into reality in health services [7]. On the transmission of FMD, various methods have been employed. Different models of the transmission of FMD have been studied. There is a lack of any persistent analytical solution for the nonlinear initial value problems (IVPs) [9-12]. These methods are less attractive and less reliable because the causes mentioned above connected with other schemes are numerically unstable.

The Euler-Maruyama, stochastic Runge-Kutta, and stochastic Euler fail to protect these dynamical characteristics of the initial system. Thus, the question arises: Does there exist a numerical technique that protects all dynamical properties [15]? Thus, this writing is an attempt to construct a reliable technique, known as stochastic nonstandard finite difference technique, for the FMD model under rules defined by Mickens in the stochastic case [16-19].

There are further sub-divisions of this paper as follows:

The deterministic FMD model and its equilibria are discussed in Sect. 2. The construction of a stochastic FMD model is discussed in Sect. 3. The comparison is drawn between 


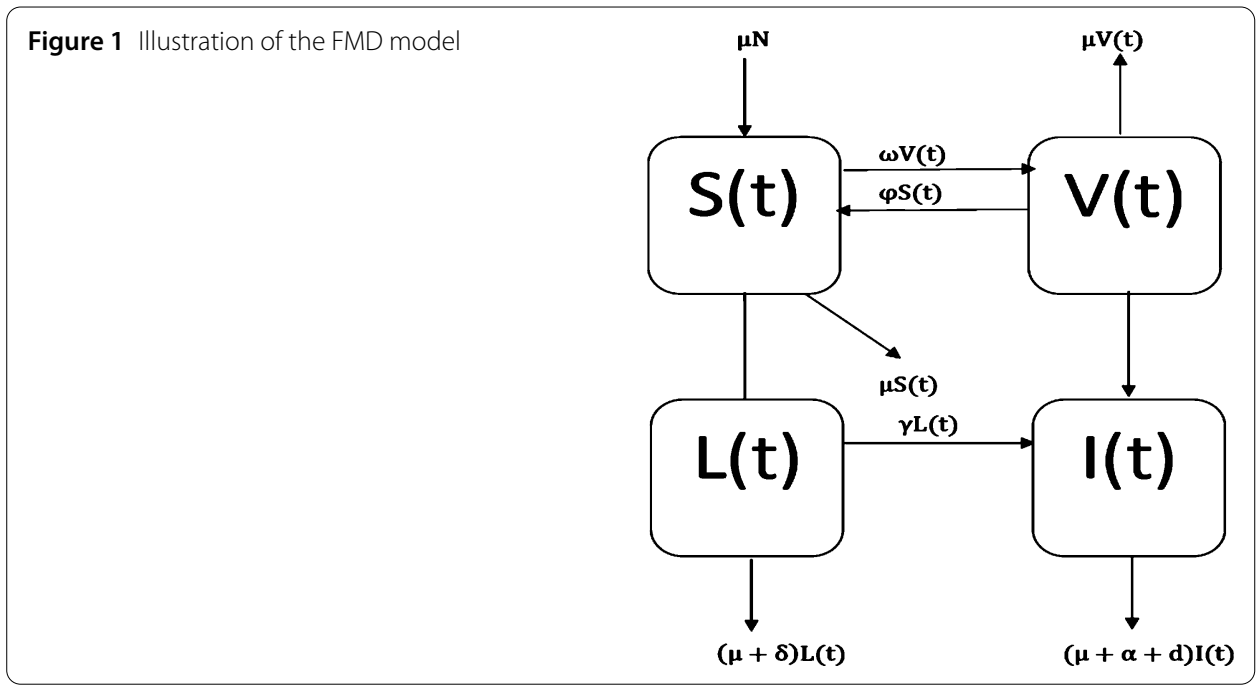

the stochastic solutions and deterministic solutions in Sect. 4. Finally, the conclusion and some future directions are given in Sect. 5 .

\section{Deterministic FMD model}

The deterministic FMD model, which is presented in [1], will be discussed in this section. Let at any arbitrary time the variables be stated as follows: $S(t)$ represents the susceptible group, $V(t)$ represents the vaccinated group, $L(t)$ represents the latently infected group, and $I(t)$ represents the infectious group, whereas $N(t)$ represents the population in Fig. 1. The flow of the FMD model is as follows.

Here, $\mu$ denotes the birth and death rate of susceptible animals, $\beta$ denotes the bilinear mass action, $\omega$ denotes the rate of vaccine wanes, $\emptyset$ denotes the vaccinated susceptible animals, $\delta$ denotes the infected animals, $\gamma$ denotes the rate of progression from the latent stage to the infection stage, $\alpha$ denotes the culled infectious animals, $d$ denotes the additional disease-related mortality.

The deterministic equations of the FMD model are as follows:

$$
\left.\begin{array}{l}
\frac{d S}{d t}=\mu N-\beta I S-(\mu+\omega) S+\emptyset V \\
\frac{d V}{d t}=\omega S-(\mu+\emptyset) V \\
\frac{d L}{d t}=\beta I S-(\mu+\delta+\gamma) L \\
\frac{d I}{d t}=\gamma L-(\mu+\alpha+d) I
\end{array}\right\}
$$

where

$$
S \geq 0, V \geq 0, L \geq 0, I \geq 0, \quad S+V+L+I \leq N .
$$

\subsection{Equilibria of FMD model}

Model (2.1) has two equilibria as follows:

Disease-free equilibrium (DFE) $D_{0}=\left(S^{0}, V^{0}, L^{0}, I^{0}\right)=\left(\frac{N(\mu+\emptyset)}{(\mu+\emptyset+\omega)}, \frac{N \omega}{(\mu+\emptyset+\omega)}, 0,0\right)$.

Endemic equilibrium (EE) $E_{1}=\left(S^{1}, V^{1}, L^{1}, I^{1}\right)$.

$$
S^{1}=\frac{N(\mu+\emptyset)}{(\mu+\emptyset+\omega) A^{*}}, \quad V^{1}=\frac{N \omega}{(\mu+\emptyset+\omega) A^{*}},
$$


Table 1 Transition of the FMD model

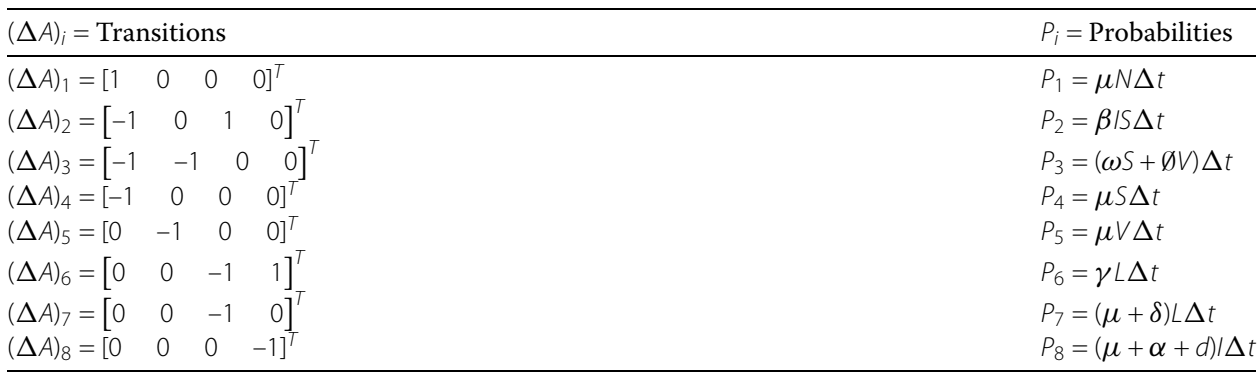

$$
L^{1}=\frac{N(\mu+\alpha+d)\left(A^{*}-1\right)}{(\mu+\alpha+\gamma+d)}, \quad I^{1}=\frac{N \gamma\left(A^{*}-1\right)}{(\mu+\alpha+\gamma+d) A^{*}}
$$

where $A^{*}=\frac{N \beta \gamma(\mu+\emptyset)}{(\alpha+d+\mu)(\gamma+\delta+\mu)(\emptyset+\omega+\mu)}$.

Note that $A^{*}$ is the FMD generation number, and it measures an average number of secondary infections occurring due to a primary infection. This number plays a decisive role in epidemiology and predicts the future of an infectious disease. If measures are taken to make this number less than 1, model (2.1) will eventually attain the disease-free equilibrium, and disease will ultimately be eradicated in an animal's population. If $A^{*}>1$, it is an alarming situation, which leads to the disease becoming endemic in an animal's population.

\section{Stochastic FMD model}

Let $A=[S, V, L, I]^{T}$ and calculate the transition probabilities of model (2.1) as follows (see Table 1):

$$
\begin{aligned}
& \text { Expectation }=\sum_{i=1}^{8} P_{i}(\Delta A)_{i}, \\
& \text { Expectation }=\left[\begin{array}{c}
\mu N-\beta I S-\omega S-\emptyset V-\mu S \\
-(\omega S+\emptyset V)-\mu V \\
\beta I S-\gamma L-(\mu+\delta) L \\
\gamma L-(\mu+\alpha+d) I
\end{array}\right] \Delta t, \\
& \left.\operatorname{Var}=\sum_{i=1}^{8} P_{i}(\Delta A)_{i}\left[(\Delta A)_{i}\right)\right]^{t},
\end{aligned}
$$

Variance

$$
\begin{aligned}
& =\left[\begin{array}{cccc}
\mu N+\beta I S+(\omega S+\emptyset V)+\mu S & (\omega S+\emptyset V) & -\beta I S & 0 \\
(\omega S+\emptyset V) & (\omega S+\emptyset V)+\mu V & 0 & 0 \\
-\beta I S & 0 & \beta I S+\gamma L+(\mu+\delta) L & -\gamma L \\
0 & 0 & -\gamma L & \gamma L+(\mu+\alpha+d) I
\end{array}\right] \Delta t, \\
& \frac{d A}{d t}=f(A, t)+L(A, t) \frac{d B}{d t} .
\end{aligned}
$$

If drift $=f(A, t)=\frac{E^{*}[\Delta A]}{\Delta t}$ and diffusion $=L(A, t)=\sqrt{\frac{E^{*}\left[\Delta A \Delta A^{T}\right]}{\Delta t}}$, then the differential equation of model (2.1) is

$$
d A=f(A, t) d t+L(A, t) d B
$$


Table 2 Parameter values

\begin{tabular}{lll}
\hline Parameters & Values (Days) & \\
\cline { 2 - 3 } & DFE & EE \\
\hline$\mu$ & 200 & 200 \\
$\gamma$ & 0.26 & 0.26 \\
$\omega$ & 0.16 & 0.16 \\
$\emptyset$ & 0.001 & 0.001 \\
$d$ & 0.001 & 0.001 \\
$\delta$ & 0.2 & 0.2 \\
$\alpha$ & 0.25 & 0.25 \\
$\beta$ & 150 & 200 \\
$N$ & 1000 & 1000 \\
$\sigma_{1}$ & 0.9 & 0.9 \\
$\sigma_{2}$ & 0.8 & 0.8 \\
$\sigma_{3}$ & 0.7 & 0.7 \\
$\sigma_{4}$ & 0.6 & 0.6 \\
\hline
\end{tabular}

with initial conditions $A(0)=A_{o}=[800,100,50,50]^{T}, 0 \leq t \leq T$, and $B$ is the Brownian motion.

\subsection{Euler-Maruyama technique}

Euler-Maruyama schemes $[14,19,20]$ are widely used to solve stochastic differential equations. We can write the Euler-Maruyama technique of SDEs (3.1) as

$$
A_{n+1}=A_{n}+f\left(A_{n}, t\right) \Delta t+L\left(A_{n}, t\right) \Delta B_{n},
$$

where ' $\Delta t$ ' represents time step size and $\Delta B_{n}$ is normally distributed in between mean and variance, i.e., $\Delta B_{n} \sim N(0,1000)$. Numerical experiments of Euler-Maruyama are performed using MATLAB taking values of parameters presented in [1], and see Table 2.

The solution of the ODEs for both equilibria is as follows: $D_{0}=(999.2,0.7994,0,0)$, and the FMD generation number $A^{*}=0.9708<1$ helps us to control this disease in the animal's population. The endemic equilibrium $E_{1}=(772,0.6176,226.9,0.2946)$ and the FMD generation number $A^{*}=1.2944>1$ show that disease is endemic in the animal's population. The graphical illustration of the aforementioned technique is shown in Fig. 2.

\section{Nonparametric perturbation in FMD model}

The idea here is to assume that each compartment has uncertainty. Thus we add the diffusion coefficient in each ODE. However, the resulting SDEs are multidimensional additive, hence there are many ways to simulate them. This idea was presented in [21, 22, 24, 28 32 ]. So, we introduce the randomness in each equation of FMD model (2.1) as follows $[2,13,24]$ :

$$
\left.\begin{array}{l}
\frac{d S}{d t}=(\mu N-\beta I S-(\mu+\omega) S-\emptyset V) d t+\sigma_{1} S d B_{1} \\
\frac{d V}{d t}=(\omega S-(\mu+\emptyset) V) d t+\sigma_{2} V d B_{2} \\
\frac{d L}{d t}=(\beta I S-(\mu+\delta+\gamma) L) d t+\sigma_{3} L d B_{3} \\
\frac{d I}{d t}=(\gamma L-(\mu+\alpha+d) I)+\sigma_{4} I d B_{4}
\end{array}\right\},
$$

where $\sigma_{1}, \sigma_{2}, \sigma_{3}$, and $\sigma_{4}$ are stochasticity of each compartment of the FMD model and $B_{j},(j=1,2,3,4$,$) are the independent Brownian motions [23, 24]. The stochastic FMD$ model (4.1) involves non-differentiability terms of Brownian motion and cannot be solved with usual numerical integrators. 


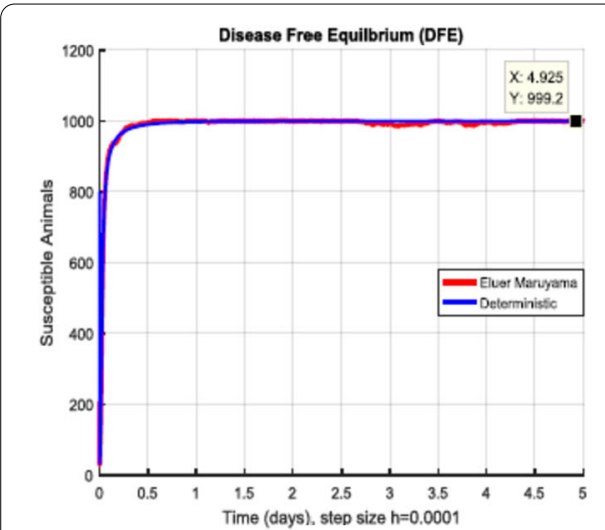

(a)

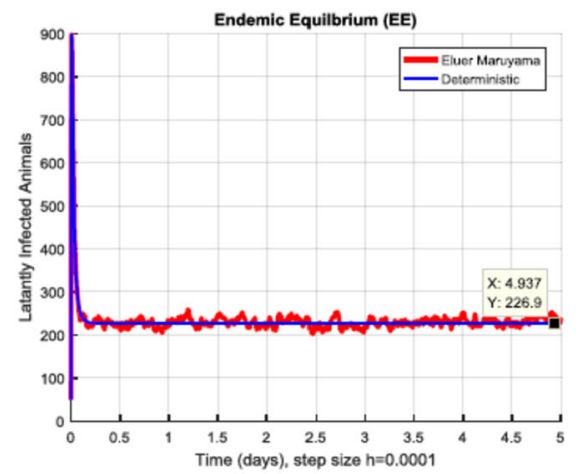

(c)

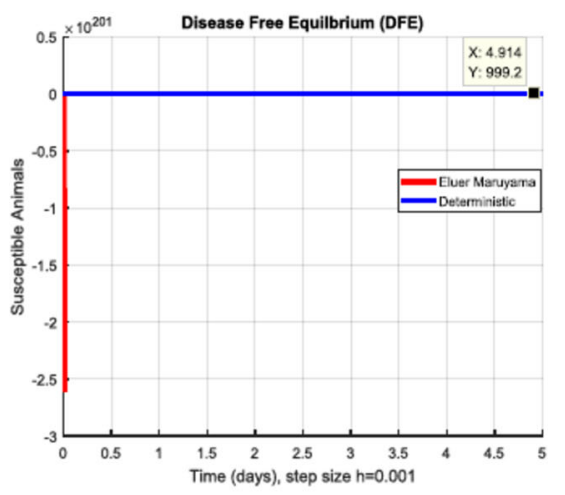

(b)

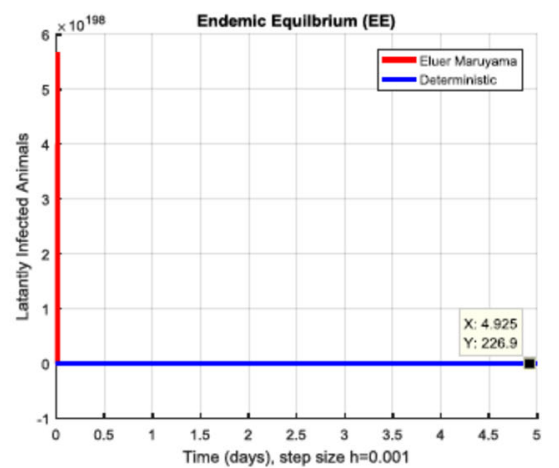

(d)

Figure 2 (a) Susceptible animals converge to disease free equilibria at $h=0.0001$. (b) Susceptible animals diverge to disease free equilibria at $h=0.001$. (c) Infectious animals converge to endemic equilibria at $h=0.0001$. (d) Infectious animals diverge to endemic equilibria at $h=0.001$

\subsection{Stochastic Euler technique}

The given technique discussed in $[2,13]$ could be extended to FMD model $(4.1)$ as follows:

$$
\left.\begin{array}{l}
S^{n+1}=S^{n}+h\left(\mu N-\beta I^{n} S^{n}-(\mu+\omega) S^{n}-\emptyset V^{n}\right)+\sigma_{1} S^{n} \Delta B_{1} \\
V^{n+1}=V^{n}+h\left(\omega S^{n}-(\mu+\emptyset) V^{n}\right)+\sigma_{2} V^{n} \Delta B_{2} \\
L^{n+1}=L^{n}+h\left(\beta I^{n} S^{n}-(\mu+\delta+\gamma) L^{n}\right)+\sigma_{3} L^{n} \Delta B_{3} \\
I^{n+1}=I^{n}+h\left(\gamma L^{n}-(\mu+\alpha+d) I^{n}\right)+\sigma_{4} I^{n} \Delta B_{4}
\end{array}\right\}
$$

Here, $h$ represents time step size and $\Delta B_{n}: n=1,2,3,4$. We use MATLAB software to simulate system (4.2) and use parameters given in [1] as presented in Fig. 3.

\subsection{Stochastic Runge-Kutta technique}

The given technique discussed in $[2,13]$ could be extended to model $(4.1)$ as follows:

First stage

$$
\begin{aligned}
& A_{1}=h\left(\mu N-\beta I^{n} S^{n}-(\mu+\omega) S^{n}-\emptyset V^{n}\right)+\sigma_{1} S^{n} \Delta B_{1}, \\
& B_{1}=h\left(\omega S^{n}-(\mu+\emptyset) V^{n}\right)+\sigma_{2} V^{n} \Delta B_{2}, \\
& C_{1}=h\left(\beta I^{n} S^{n}-(\mu+\delta+\gamma) L^{n}\right)+\sigma_{3} L^{n} \Delta B_{3}, \\
& D_{1}=h\left(\gamma L^{n}-(\mu+\alpha+d) I^{n}\right)+\sigma_{4} I^{n} \Delta B_{4} .
\end{aligned}
$$




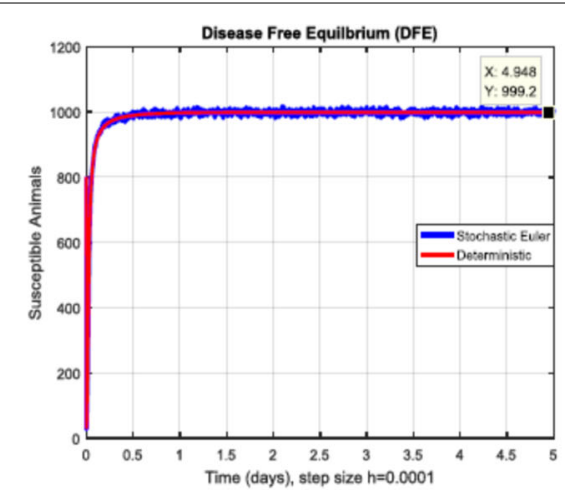

(a)

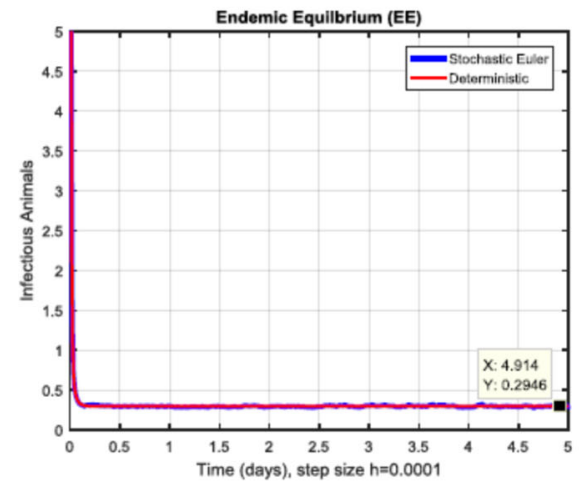

(c)

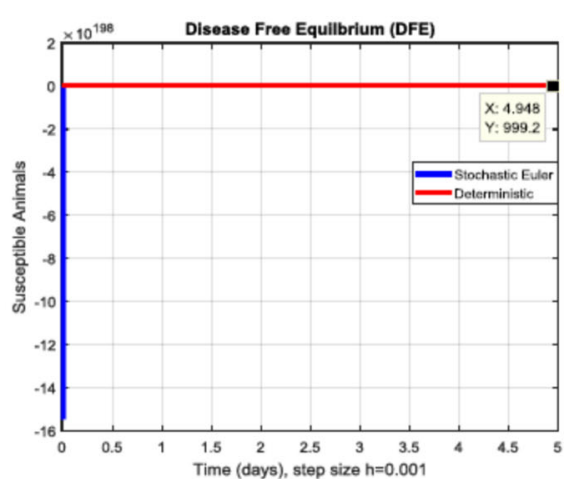

(b)

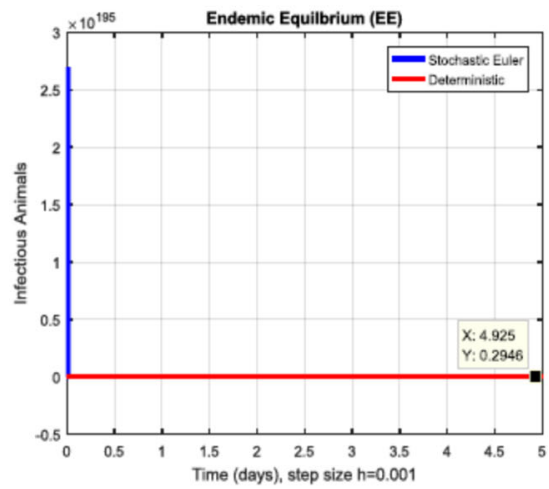

(d)

Figure 3 (a) Susceptible animals converge to disease free equilibria at $h=0.0001$. (b) Susceptible animals diverge to disease free equilibria at $h=0.001$. (c) Infectious animals converge to endemic equilibria at $h=0.0001$. (d) Infectious animals diverge to endemic equilibria at $h=0.001$

\section{Second stage}

$$
\begin{aligned}
A_{2}= & h\left(\mu N-\beta\left(I^{n}+\frac{D_{1}}{2}\right)\left(S^{n}+\frac{A_{1}}{2}\right)-(\mu+\omega)\left(S^{n}+\frac{A_{1}}{2}\right)\right. \\
& \left.-\emptyset\left(V^{n} \frac{B_{1}}{2}\right)\right)+\sigma_{1}\left(S^{n}+\frac{A_{1}}{2}\right) \Delta B_{1}, \\
B_{2}= & h\left(\omega\left(S^{n}+\frac{A_{1}}{2}\right)-(\mu+\emptyset)\left(V^{n}+\frac{B_{1}}{2}\right)+\sigma_{2}\left(V^{n}+\frac{B_{1}}{2}\right) \Delta B_{2},\right. \\
C_{2}= & h\left(\beta\left(I^{n}+\frac{D_{1}}{2}\right)\left(S^{n}+\frac{A_{1}}{2}\right)-(\mu+\delta+\gamma)\left(L^{n}+\frac{C_{1}}{2}\right)\right)+\sigma_{3}\left(L^{n}+\frac{C_{1}}{2}\right) \Delta B_{3}, \\
D_{2}= & h\left(\gamma\left(L^{n}+\frac{C_{1}}{2}\right)-(\mu+\alpha+d)\left(I^{n}+\frac{D_{1}}{2}\right)\right)+\sigma_{4}\left(I^{n}+\frac{D_{1}}{2}\right) \Delta B_{4} .
\end{aligned}
$$

Third stage

$$
\begin{aligned}
A_{3}= & h\left(\mu N-\beta\left(I^{n}+\frac{D_{2}}{2}\right)\left(S^{n}+\frac{A_{2}}{2}\right)-(\mu+\omega)\left(S^{n}+\frac{A_{2}}{2}\right)-\emptyset\left(V^{n} \frac{B_{2}}{2}\right)\right) \\
& +\sigma_{1}\left(S^{n}+\frac{A_{2}}{2}\right) \Delta B_{1},
\end{aligned}
$$




$$
\begin{aligned}
& B_{3}=h\left(\omega\left(S^{n}+\frac{A_{2}}{2}\right)-(\mu+\emptyset)\left(V^{n}+\frac{B_{2}}{2}\right)+\sigma_{2}\left(V^{n}+\frac{B_{2}}{2}\right) \Delta B_{2},\right. \\
& C_{3}=h\left(\beta\left(I^{n}+\frac{D_{2}}{2}\right)\left(S^{n}+\frac{A_{2}}{2}\right)-(\mu+\delta+\gamma)\left(L^{n}+\frac{C_{2}}{2}\right)\right)+\sigma_{3}\left(L^{n}+\frac{C_{2}}{2}\right) \Delta B_{3}, \\
& D_{3}=h\left(\gamma\left(L^{n}+\frac{C_{2}}{2}\right)-(\mu+\alpha+d)\left(I^{n}+\frac{D_{2}}{2}\right)\right)+\sigma_{4}\left(I^{n}+\frac{D_{2}}{2}\right) \Delta B_{4} .
\end{aligned}
$$

Fourth stage

$$
\begin{aligned}
A_{4}= & h\left(\mu N-\beta\left(I^{n}+\frac{D_{3}}{2}\right)\left(S^{n}+\frac{A_{3}}{2}\right)-(\mu+\omega)\left(S^{n}+\frac{A_{3}}{2}\right)-\emptyset\left(V^{n} \frac{B_{3}}{2}\right)\right) \\
& +\sigma_{1}\left(S^{n}+\frac{A_{3}}{2}\right) \Delta B_{1}, \\
B_{4}= & h\left(\omega\left(S^{n}+\frac{A_{3}}{2}\right)-(\mu+\emptyset)\left(V^{n}+\frac{B_{3}}{2}\right)+\sigma_{2}\left(V^{n}+\frac{B_{3}}{2}\right) \Delta B_{2},\right. \\
C_{4}= & h\left(\beta\left(I^{n}+\frac{D_{3}}{2}\right)\left(S^{n}+\frac{A_{3}}{2}\right)-(\mu+\delta+\gamma)\left(L^{n}+\frac{C_{3}}{2}\right)\right)+\sigma_{3}\left(L^{n}+\frac{C_{3}}{2}\right) \Delta B_{3}, \\
D_{4}= & h\left(\gamma\left(L^{n}+\frac{C_{3}}{2}\right)-(\mu+\alpha+d)\left(I^{n}+\frac{D_{3}}{2}\right)\right)+\sigma_{4}\left(I^{n}+\frac{D_{3}}{2}\right) \Delta B_{4} .
\end{aligned}
$$

Final stage

$$
\left.\begin{array}{l}
S^{n+1}=S^{n}+\frac{1}{6}\left[A_{1}+2 A_{2}+2 A_{3}+A_{4}\right] \\
V^{n+1}=V^{n}+\frac{1}{6}\left[B_{1}+2 B_{2}+2 B_{3}+B_{4}\right] \\
L^{n+1}=L^{n}+\frac{1}{6}\left[C_{1}+2 C_{2}+2 C_{3}+C_{4}\right] \\
I^{n+1}=I^{n}+\frac{1}{6}\left[D_{1}+2 D_{2}+2 D_{3}+D_{4}\right]
\end{array}\right\}
$$

Here, $h$ represents time step size and $\Delta B_{n}: n=1,2,3,4$. System (4.3) is simulated by using MATLAB, taking parameters from [1].

\subsection{Stochastic NSFD technique}

The stochastic nonstandard finite difference technique (SNSFD) is constructed with the aim that it preserves all the dynamical properties defined by Mickens in a stochastic context. Now, we extend our proposed SNSFD technique $[2,13]$ to model $(4.1)$ as follows:

$$
\left.\begin{array}{l}
S^{n+1}=\frac{S^{n}+h \mu N+h \sigma_{1} S^{n} \Delta B_{1}}{1+h \beta_{1} I^{n}+h(\mu+\omega)} \\
V^{n+1}=\frac{h \omega S^{n}+\sigma_{2} V^{n} \Delta B_{2}}{1+h(\mu+\emptyset)} \\
L^{n+1}=\frac{L^{n}+h \beta I^{n} S^{n}+h \sigma_{3} L^{n} \Delta B_{3}}{1+h(\mu+\delta+\gamma)} \\
I^{n+1}=\frac{I^{n}+h \gamma L^{n}+h \sigma_{4} I^{n} \Delta B_{4}}{1+h(\mu+\alpha+d)}
\end{array}\right\} .
$$

Here, $h$ represents time step size and $\Delta B_{n}: n=1,2,3,4$. System (4.4) is simulated by using MATLAB taking parameters from [1].

\subsubsection{Stability analysis}

The following theorems are presented in support of the given analysis.

Theorem 4.1 The disease-free point with $A^{*}<1$ and endemic point with $A^{*}>1$ are stable, then the absolute eigenvalues of the discrete model on both equilibria lie in a unit circle [16]. 
Proof We consider the newly constructed SNSFD method as follows:

$$
\begin{aligned}
& F=\frac{S+h \mu N+h \emptyset V+h \sigma_{1} S \Delta B_{1}}{1+h \beta I+h(\mu+\omega)}, \\
& G=\frac{V+h \omega S+h \sigma_{2} V \Delta B_{2}}{1+h(\mu+\emptyset)}, \\
& H=\frac{L+h \beta I S+h \sigma_{3} L \Delta B_{3}}{1+h(\mu+\delta+\gamma)}, \\
& R=\frac{I+h \gamma L+h \sigma_{4} I \Delta B_{4}}{1+h(\mu+\alpha+d)}, \\
& \frac{\partial F}{\partial S}=\frac{1+h \sigma_{1} \Delta B_{1}}{1+h \beta I+h(\mu+\omega)}, \quad \frac{\partial F}{\partial L}=0, \quad \frac{\partial F}{\partial V}=\frac{h \emptyset}{1+h \beta I+h(\mu+\omega)}, \\
& \frac{\partial F}{\partial I}=\frac{-\left(S+h \mu N+h \emptyset V+h \sigma_{1} S \Delta B_{1}\right)(h \beta)}{(1+h \beta I+h(\mu+\omega))^{2}}, \\
& \frac{\partial G}{\partial S}=\frac{h \omega}{1+h(\mu+\emptyset)}, \quad \frac{\partial G}{\partial L}=0, \\
& \frac{\partial H}{\partial S}=\frac{h G}{1+h(\mu+\delta+\gamma)}, \quad \frac{\partial H}{\partial L}=\frac{1+h \sigma_{2} \Delta B_{2}}{1+h\left(\mu+\delta \sigma_{3} \Delta B_{3}\right.}, \quad \frac{\partial G}{\partial I}=0, \\
& \frac{\partial H}{\partial V}=0, \quad \frac{\partial H}{\partial I}=\frac{h+\gamma)}{1+h(\mu+\delta+\gamma)}, \\
& \frac{\partial K}{\partial S}=0, \quad \frac{\partial K}{\partial V}=0, \quad \frac{\partial K}{\partial L}=\frac{h \gamma}{1+h(\mu+\alpha+d)}, \quad \frac{\partial K}{\partial I}=\frac{1+h \sigma_{4} \Delta B_{4}}{1+h(\mu+\alpha+d)} .
\end{aligned}
$$

The Jacobean matrix as follows:

$$
\begin{aligned}
J & =\left[\begin{array}{llll}
\frac{\partial F}{\partial S} & \frac{\partial F}{\partial V} & \frac{\partial F}{\partial L} & \frac{\partial F}{\partial I} \\
\frac{\partial G}{\partial S} & \frac{\partial G}{\partial V} & \frac{\partial G}{\partial L} & \frac{\partial G}{\partial I} \\
\frac{\partial H}{\partial S} & \frac{\partial H}{\partial V} & \frac{\partial H}{\partial L} & \frac{\partial H}{\partial I} \\
\frac{\partial K}{\partial S} & \frac{\partial K}{\partial V} & \frac{\partial K}{\partial L} & \frac{\partial K}{\partial I}
\end{array}\right], \\
J & =\left[\begin{array}{cccc}
\frac{1+h \sigma_{1} \Delta B_{1}}{1+h \beta I+h(\mu+\omega)} & \frac{h \emptyset}{1+h \beta I+h(\mu+\omega)} & 0 & \frac{-\left(S+h \mu N+h \emptyset V+h \sigma_{1} S \Delta B_{1}\right)(h \beta)}{(1+h \beta I+h(\mu+\omega))^{2}} \\
\frac{h \omega}{1+h(\mu+\emptyset)} & \frac{1+h \Delta B_{2}}{1+h(\mu+\varnothing)} & 0 & 0 \\
\frac{h \beta I}{1+h(\mu+\delta+\gamma)} & 0 & \frac{1+h \sigma_{3} \Delta B_{3}}{1+h(\mu+\delta+\gamma)} & \frac{h \beta S}{1+h(\mu+\delta+\gamma)} \\
0 & 0 & \frac{h \gamma}{1+h(\mu+\alpha+d)} & \frac{1+h \sigma_{4} \Delta B_{4}}{1+h(\mu+\alpha+d)}
\end{array}\right] .
\end{aligned}
$$

So, by using MATLAB software, we have plotted the dominant eigenvalue of the given Jacobean matrix $J$ as shown in Fig. 6.

Remarks For both equilibria, the largest eigenvalue is less than one. Ultimately, the other three eigenvalues for both of these cases are also less than one. So, the given technique stochastic nonstandard finite difference for the discrete dynamical system is locally asymptotical stable (LAS) for both equilibria.

\subsection{Contrast section}

In this section, we shall make a comparison among stochastic explicit numerical methods and stochastic NSFD as follows. 


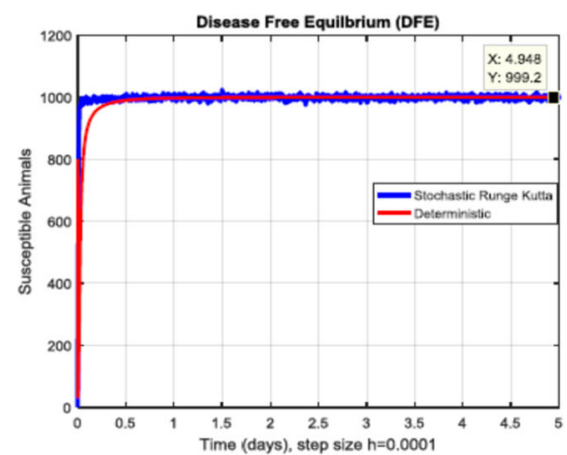

(a)

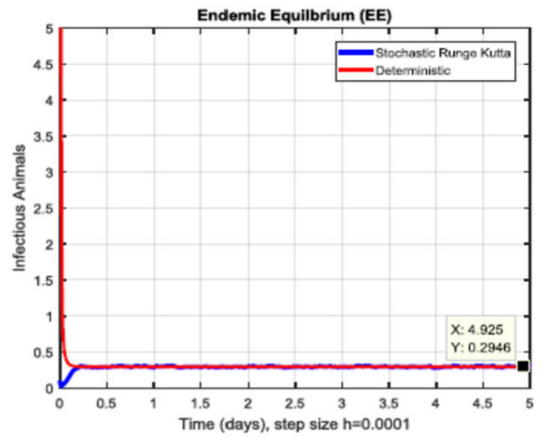

(c)

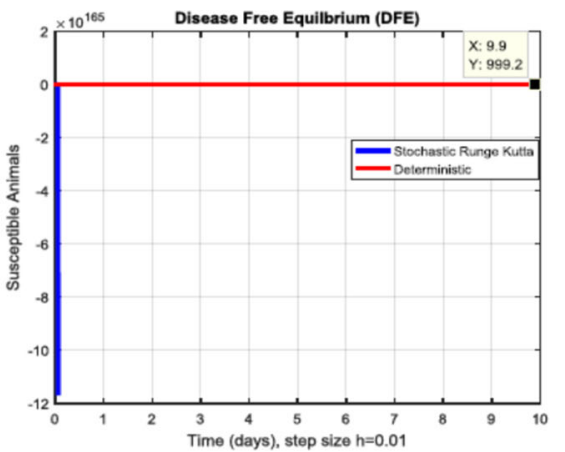

(b)

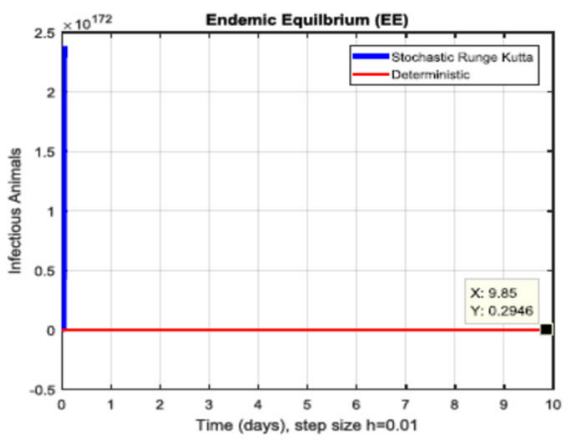

(d)

Figure 4 (a) Susceptible animals converge to disease free equilibria at $h=0.0001$. (b) Susceptible animals diverge to disease free equilibria at $h=0.01$. (c) Infectious animals converge to endemic equilibria at $h=0.0001$. (d) Infectious animals diverge to endemic equilibria at $h=0.01$

\section{Consequences and discussion}

The Euler-Maruyama scheme meets the actual equilibria of the FMD model and illustrates that a deterministic outcome is the mean of the Euler-Maruyama outcome in Fig. 2(a) and Fig. 2(c). However, the given technique is divergent at the increase in time step size, as shown in Fig. 2(b) and Fig. 2(d). The stochastic Euler technique meets the factual equilibria, and the deterministic outcome is its mean as shown in Fig. 3(a) and Fig. 3(c). This technique is also a time-dependent technique as shown in Fig. 3(b) and Fig. 3(d). The stochastic RK technique approaches to true equilibria of the model as shown in Fig. 4(a) and (c). The stochastic RK technique is also time-dependent, as shown in Fig. 4(b) and (d). The stochastic nonstandard finite difference technique approaches to true equilibria of the model at any value of parameter as shown in Fig. 5. The proposed technique is a time-independent technique. The largest eigenvalue has been plotted for both equilibria as shown in Fig. 6(a) and Fig. 6(b).

We have also presented a comparison of these methods in Fig. 7. The comparison of Euler-Maruyama and stochastic NSFD for DFE and EE is shown in Fig. 7(a) and Fig. 7(b). The comparison of stochastic Euler and stochastic NSFD for both DFE and EE is shown in Fig. 7(c) and Fig. 7(d). The comparison of stochastic Runge-Kutta and stochastic NSFD for both DFE and EE is shown in Fig. 7(e) and Fig. 7(f). Thus we concluded that the stochastic explicit numerical techniques do not preserve the dynamical properties presented in [1618] as shown in Fig. 7(b), Fig. 7(d) and Fig. 7(f). 


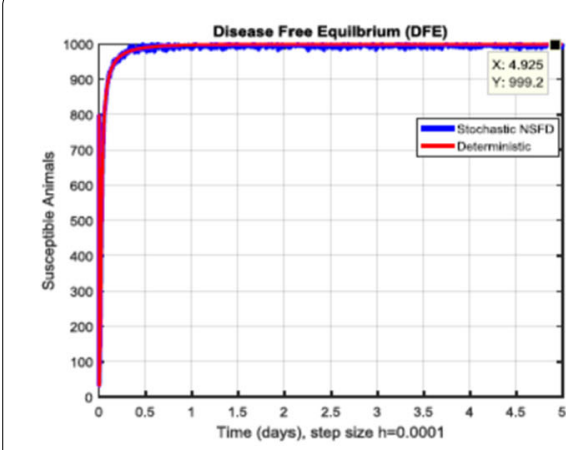

(a)

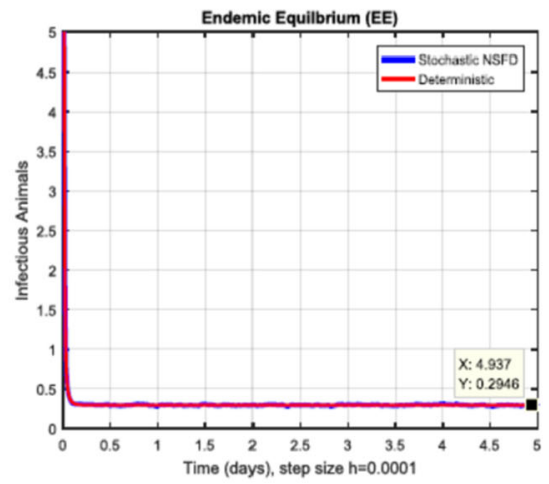

(c)

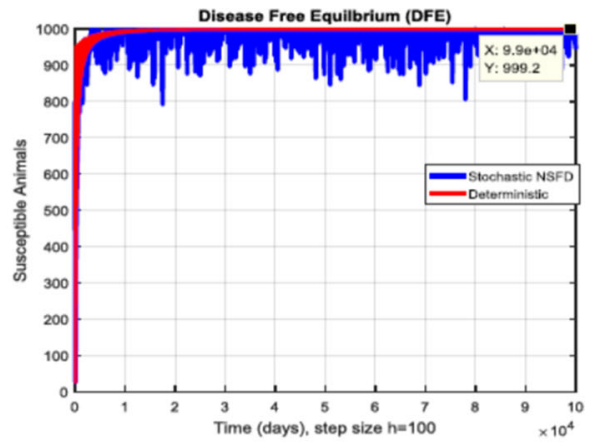

(b)

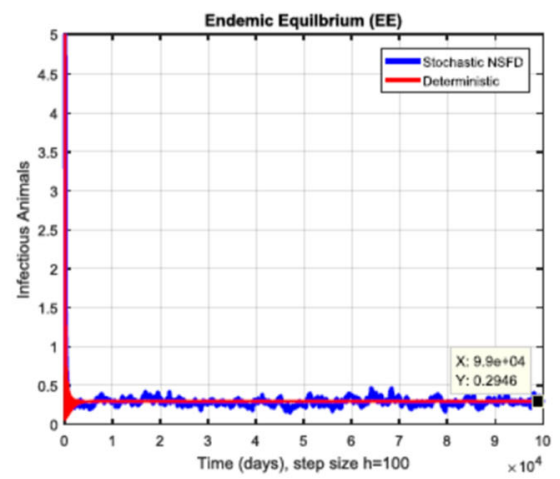

(d)

Figure 5 (a) Susceptible animals converge to disease free equilibria at $h=0.0001$. (b) Susceptible animals converges to disease free equilibria at $h=100$. (c) Infectious animals converges to endemic equilibria at $h=0.0001$. (d) Infectious animals converges to endemic equilibria at $h=100$. It means that stochastic nonstandard finite difference scheme (SNSFD) is always convergent to equilibria of model at any time step size parameter. This is the beauty of SNSFD because independent of time step size

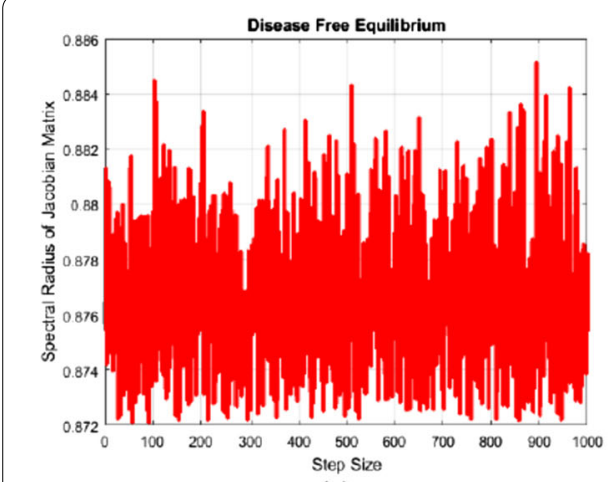

(a)

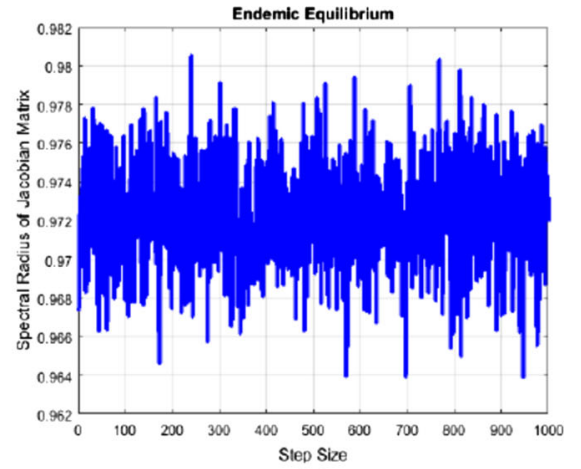

(b)

Figure 6 (a) Spectral Radius for DFE. (b) Spectral Radius for EE

\section{Conclusion and directions}

The deterministic epidemic model is a less reliable technique as compared to the stochastic model in terms of numerical analysis for comprehending FMD model dynamics. The numerical techniques which are discussed above approach to equilibria of the model for very small step size. In the stochastic framework our proposed technique of SNSFD for the 


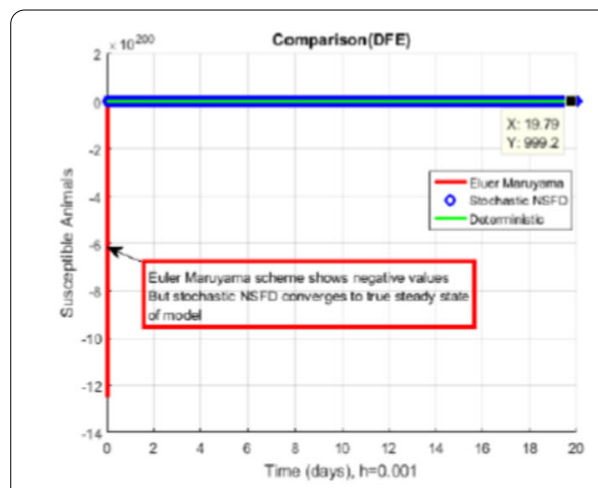

(a)

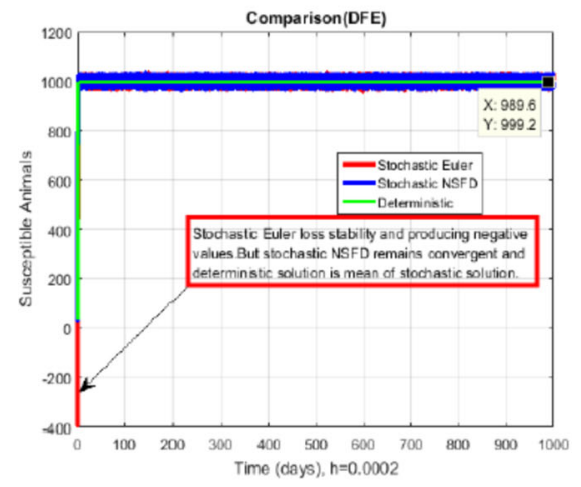

(c)

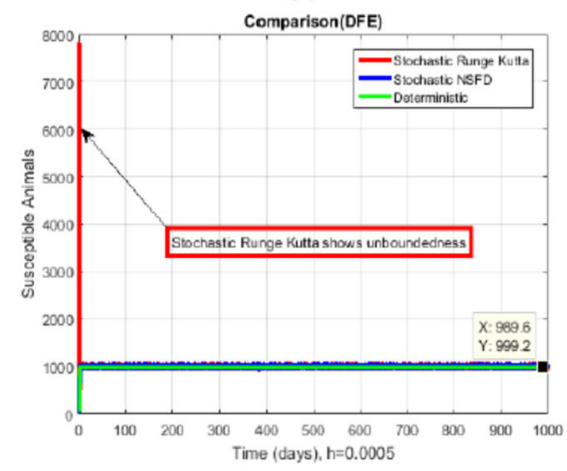

(e)

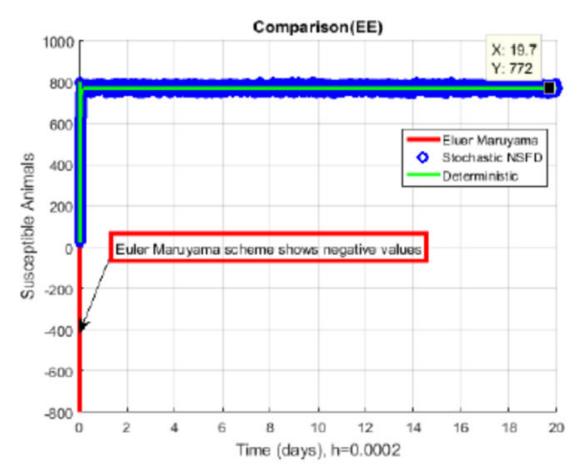

(b)

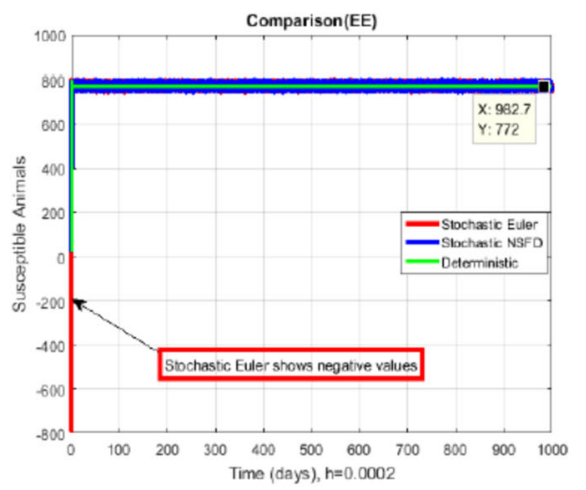

(d)

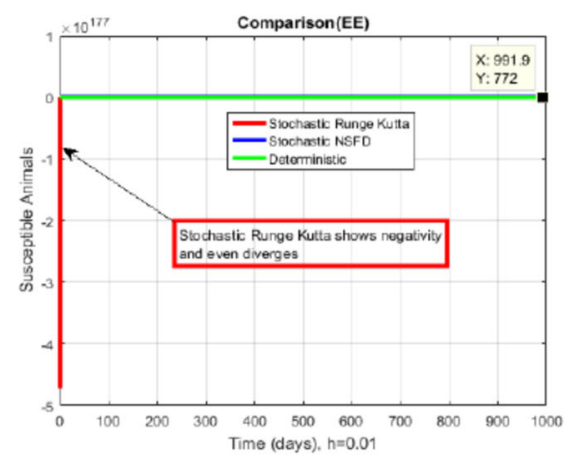

(f)

Figure 7 Comparison in solutions of explicit methods, stochastic NSFD and deterministic

FMD model performs for any time step size $[17,18]$. In disease dynamics the stochastic modelling plays a significant role. We have observed that the SNSFD is the most appropriate technique to tackle all complex stochastic models. It is also noted that deterministic epidemic models are less pragmatic as compared to stochastic epidemic models. The complicated stochastic diffusion and delay models can be studied by applying the stochastic nonstandard finite difference technique for future work. We can extend this analysis to the fractional-order dynamical system [25-27]. 
environment and facilities. The first author thanks Prince Sultan University for supporting this paper through the research group Nonlinear Analysis Methods in Applied Mathematics (NAMAM), group number RG-DES-2017-01-17.

\section{Funding}

We have no funding for this article.

\section{Abbreviations}

FMD, Foot and Mouth Disease; SDE, Stochastic Differential Equation; SNSFD, Stochastic Non-Standard Finite-Difference.

\section{Competing interests}

The authors declare that they have no competing interests.

\section{Authors' contributions}

We have an equal contribution, and careful reading has been done from all authors.

\section{Author details}

${ }^{1}$ Department of Mathematics and General Sciences, Prince Sultan University, Riyadh, Saudi Arabia. ${ }^{2}$ Stochastic Analysis \& Optimization Resaerch Group, Department of Mathematics, Air University PAF Complex E-9, Islamabad, Pakistan. ${ }^{3}$ Faculty of Engineering, University of Central Punjab, Lahore, Pakistan. ${ }^{4}$ Department of Mathematics, Chak Shahzad Campus, Comsats University, Islamabad, Pakistan.

\section{Publisher's Note}

Springer Nature remains neutral with regard to jurisdictional claims in published maps and institutional affiliations.

\section{Received: 3 October 2019 Accepted: 8 January 2020 Published online: 16 January 2020}

\section{References}

1. Mushayabasa, S., Bhunu, C.P., Dhlamini, M.: Impact of vaccination and culling on controlling foot and mouth disease a mathematical modelling approach. World J. Vaccines 1, 156-161 (2011)

2. Raza, A., Arif, M.S., Rafiq, M.: A reliable numerical analysis for stochastic dengue epidemic model with incubation period of virus. Adv. Differ. Equ. 2019, 32 (2019)

3. Marcos, A., Perez, A.M.: Quantitative risk assessment of foot and mouth disease (FMD) virus introduction into the FMD free zone without vaccination of Argentina through legal and illegal trade of bone in beef and unvaccinated susceptible species. Front. Vet. Sci. 6, 78 (2019)

4. Alvarez, J., Bakker, D., Bezos, J.: Epidemiology and control of notifiable animals' diseases. Front. Vet. Sci. 6, 43 (2019)

5. Schley, D., Ward, J., Zhang, Z: Modelling foot and mouth disease virus dynamics in oral epithelium to help identify the determinants of lysis. Bull. Math. Biol. 73, 1503-1528 (2019)

6. Tildesley, M.J., Keeling, M.J.: Modelling foot and mouth disease a comparison between the UK and Denmark. Prev. Vet. Med. 85, 107-124 (2008)

7. Kitching, R.P., Hutber, A.M., Thrusfield, M.V.: A review of foot and mouth disease with special consideration for the clinical and epidemiological factors relevant to predictive modelling of the disease. Vet. J. 169, 197-209 (2005)

8. Armstrong, R.M., Mathew, E.S.: Predicting herd protection against foot and mouth disease by testing individual and bulk milk samples. J. Virol. Methods 97, 87-99 (2001)

9. Raza, A., Arif, M.S., Rafiq, M.: A reliable numerical analysis for stochastic gonorrhea epidemic model with treatment effect. Int. J. Biomath. 12, 01 (2019)

10. Zafar, Z., Rehan, K., Mushtaq, M., et al.: Numerical treatment for nonlinear brusselator chemical model. J. Differ. Equ. Appl. 23(3), 521-538 (2017)

11. Arif, M.S., Raza, A., Rafiq, M., et al.: A reliable numerical analysis for stochastic hepatitis B virus epidemic model with the migration effect. Iran. J. Sci. Technol., Trans. A, Sci. 43, 2477-2492 (2019)

12. Zafar, Z., Rehan, K., Mushtaq, M.: HIV/AIDS epidemic fractional-order model. J. Differ. Equ. Appl. 23(7), 1298-1315 (2017)

13. Arif, M.S., Raza, A., Rafiq, M., et al.: A reliable stochastic numerical analysis for typhoid fever incorporating with protection against infection. Comput. Mater. Continua 59(3), 787-804 (2019)

14. Oksendal, B:: Stochastic Differential Equations. Springer, Berlin (2003)

15. Cresson, J., Pierret, F.: Nonstandard finite difference scheme preserving dynamical properties (2014). Preprint arXiv:1410.6661

16. Mickens, R.E.: Nonstandard Finite Difference Models of Differential Equations. World Scientific, River Edge (1994)

17. Mickens, R.E.: Advances in Applications of Nonstandard Finite Difference Schemes. World Scientific, Hackensack (2005)

18. Mickens, R.E.: A fundamental principle for constructing nonstandard finite difference schemes for differential equations. J. Differ. Equ. Appl. 11(7), 645-653 (2005)

19. Gard, T.C.: Introduction to Stochastic Differential Equations. Dekker, New York (1988)

20. Karatzas, I., Shreve, S.E.: Brownian Motion and Stochastic Calculus, 2nd edn. Springer, Berlin (1991)

21. Allen, L.J.S., Burgin, A.: Comparison of deterministic and stochastic SIS and SIR models in discrete time. Math. Biosci. $163,1-33(2000)$

22. Allen, E.J.: Modeling with Ito Stochastic Differential Equations. Springer, Dordrecht (2007)

23. Britton, T: Stochastic epidemic models. Math. Biosci. 225(1), 24-35 (2010)

24. Allen, E.J., Allen, L.J.S., Arciniega, A., et al.: Construction of equivalent stochastic differential equation models. Stoch. Anal. Appl. 26(2), 274-297 (2008)

25. Jajarmi, A., Baleanu, D.: A new fractional analysis on the interaction of HIV with CD4+ T-cells. Chaos Solitons Fractals $113,221-229(2018)$ 
26. Baleanu, D., Jajarmi, A., Bonyah, E., et al.: New aspects of poor nutrition in the life cycle within the fractional calculus. Adv. Differ. Equ. 2018, 230 (2018)

27. Baleanu, D., Doha, E.H., Abdelkawy, M.A., et al.: Approximate solutions for solving nonlinear variable-order fractional Riccati differential equations. Nonlinear Anal., Model. Control 24, 176-188 (2019)

28. Heyman, D.P., Sobel, M.J.: Stochastic Models, 1st edn. North-Holland, Amsterdam (1990)

29. Tijms, H.C.: Stochastic Models: An Algorithmic Approach, 1st edn. Wiley, New York (1995)

30. Iosifescu, M., Limnios, N., Oprisan, G.: Introduction to Stochastic Models, 1st edn. Wiley, New York (2010)

31. Nelson, B.L: Stochastic Modeling Analysis and Simulation. McGraw Hills, New York (2010)

32. Nicolas, L.: Stochastic Modeling. Springer, Berlin (2017)

Submit your manuscript to a SpringerOpen ${ }^{\circ}$ journal and benefit from:

- Convenient online submission

- Rigorous peer review

Open access: articles freely available online

- High visibility within the field

- Retaining the copyright to your article

Submit your next manuscript at $\gg$ springeropen.com 